\title{
Impacts of Voltage-based Grid Support Functions on Energy Production of PV Customers
}

\author{
Julieta Giraldez, Michael Emmanuel, Andy Hoke \\ National Renewable Energy Laboratory
}

Siddharth Suryanarayanan

Colorado State University

\section{Introduction}

Increasing levels of Distributed Energy Resources (DERs) located at or close to the customer site is changing the way the power system, and in particular the distribution system, is planned and operated. At the distribution level, distribution utilities are looking at the impact of voltage-based GSFs like Volt/VAR and Volt/Watt in voltage regulating strategies, as well as in customers' energy curtailment values that are activating such functions.

Our study proposes four metrics---maximum GSF and average GSF curtailment, average increased generation and average net generation change---to assess the full impact of a given GSF control on customer-sited PV systems. We then apply those metrics to several detailed quasi-static time-series (QSTS) simulations of a distribution feeder with various levels of PV generation.

\section{Voltage-based Grid Support Functions, Metrics, and Impacts}

The volt/var and volt/watt curves used in the study are shown in Figure 1.
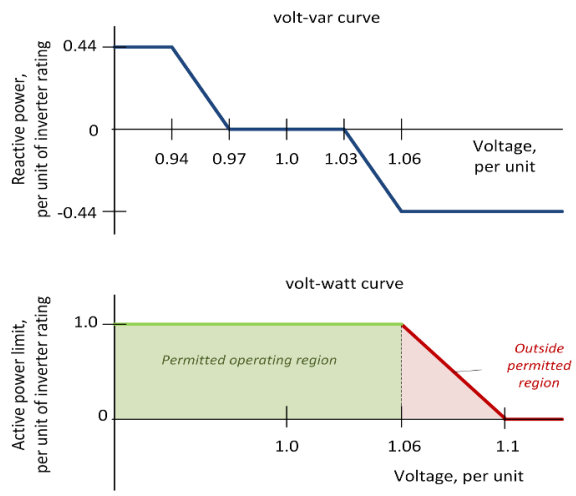

Figure 1. Volt-var and Volt-watt grid support functions and the settings used in this study

Reactive power-based functions such as Volt/Var control can be configured to prioritize either active or reactive power when the inverter's current limit does not allow it to produce the desired amount of both $\mathrm{P}$ and $\mathrm{Q}$ simultaneously.

Active power priority (Watt priority) can be used to ensure zero impact on energy production when providing reactive-power support to the grid. This was briefly called for in California Rule 21 ; however, active power priority causes grid support to be unavailable during times of high PV production, when voltage control is most needed.
The IEEE Standard 1547-2018 revision requires reactive powe priority to allow DERs to supply or absorb reactive power when available up to $44 \%$ of the nameplate KVA rating for maximum Var injection and absorption at rated DER voltage.

Enabling the Var priority mode can lead to active power curtailment as shown in (1)-(2), $P_{\text {VVar Var }}$ is:

$$
P_{\text {VVar,Var }}=\sqrt{\left(S_{\text {inv }}\right)^{2}-\left(Q_{\text {VVar.Var }}\right)^{2}}
$$

where $S_{i n v}$ and $\mathrm{Q}_{V V a r}$ are the inverter capacity (kVA) and reactive power, respectively. The Var priority mode will result in $P_{\text {Curt }}$ curtailment if the inverter capacity is not large enough to provide reactive power support based on the droop curve. The active power curtailed $P_{\text {Curt }}$ shown in Figure 2 is given as:

$$
P_{\text {Curt }}=P_{\text {VVar,Watt }}-P_{\text {VVar,Var }}
$$

where $P_{V V a r, W a t t}$ and $P_{V V a r, \text { Var }}$ are the available active power in active and reactive power priority modes respectively.

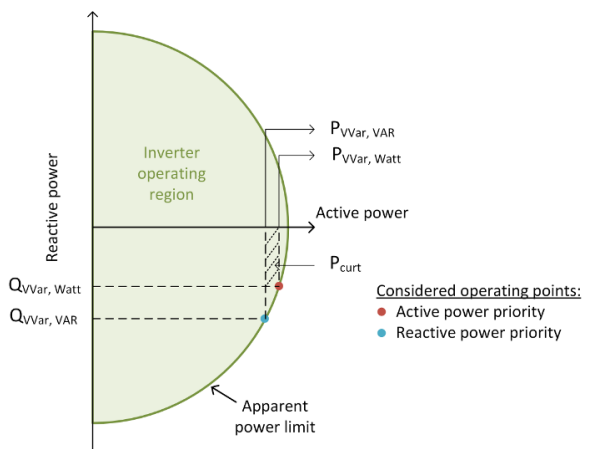

Figure 2. Volt/Var reactive power and active power priority modes

\section{Metrics}

From the perspective of the customer, voltage-based GSFs from the DERs can impact the energy production since the active power output of the inverter may be reduced during GSF actions. The metrics proposed in this paper are:

- Max GSF curtailment is the maximum customer energy curtailed over a given time period.

- Average GSF curtailment is the average customer energy curtailed over a given time period.

Average increased generation is the average custome increased energy generation at the customer site for a given time period because resulting from reduced $\mathrm{PV}$ inverte disconnections for voltages above $1.1 \mathrm{p.u}$.

- Average net generation change is the average custome increased generation minus the average grid-support function curtailment for a given time period. A positive value represents a net increase in $\mathrm{PV}$ generation.

\section{Results of high-penetration PV cases with VV and VV-VW}

The maximum customer curtailment in Volt/Var mode remains at $1.8 \%$ and is independent of the PV penetration level. However, for the Volt/Var-Volt/Watt mode, the maximum customer curtailment increases rapidly from $2.3 \%$ in the low PV penetration case to $5.7 \%$ in the high PV penetration. Yet, the average customer energy curtailment values are the same or slightly lower in the Volt/Var-Volt/Watt case, than in the Volt/Var alone scenario. This suggests that very few customers experience non-negligible Volt/Watt GSF activation, and that the effectiveness of Volt/Watt in lowering voltages for a few outlier customers slightly lowers the curtailment for the remaining of the customers that experience only Volt/Var activation.

Table 1. Impact of activating GSF control on PV systems and energy curtailment at different penetration levels

\begin{tabular}{|l|c|c|c|c|c|c|}
\hline \multirow{2}{*}{ Metrics } & \multicolumn{7}{|c|}{ GDML penetration levels } \\
\hline & \multicolumn{2}{|c|}{$175 \%$} & \multicolumn{2}{c|}{$37 \%$} & \multicolumn{2}{c|}{$600 \%$} \\
\hline Max GSF Curt. & $1.8 \%$ & $2.3 \%$ & $1.8 \%$ & $3.7 \%$ & $1.8 \%$ & $5.7 \%$ \\
\hline Ave. GSF Curt. & $0.10 \%$ & $0.07 \%$ & $0.15 \%$ & $0.13 \%$ & $0.24 \%$ & $0.23 \%$ \\
\hline Ave. Incr. Gen. & $2.1 \%$ & $2 \%$ & $2.7 \%$ & $2.6 \%$ & $2.7 \%$ & $3 \%$ \\
\hline Ave. Net Gen. & $2 \%$ & $1.9 \%$ & $2.5 \%$ & $2.4 \%$ & $2.4 \%$ & $2.8 \%$ \\
\hline
\end{tabular}

These findings are illustrated by plotting the customer energy curtailment values against the maximum voltage experienced by the customer. In the very high PV penetration case, still less than 10 customers out of 531 PV systems are affected by this activation. Energy curtailment is negligible even for the very high penetration case provided peak voltages are within the ANSI C84. 1 range.

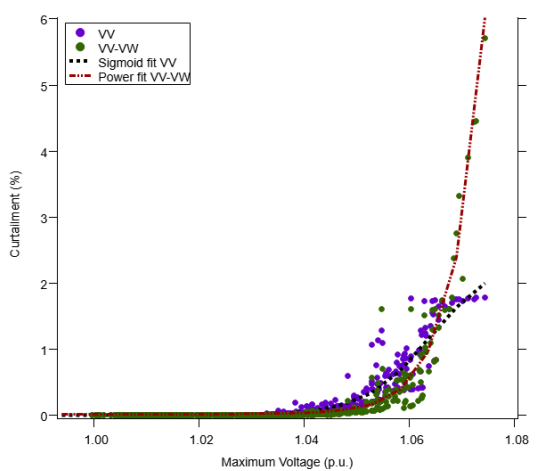

Figure 3. Weekly customer energy curtailment versus maximum voltage for the high PV penetration case

The utility can leverage this because voltage violation problems may point to possible curtailment issues, which require appropriate mitigation measures. Voltage violation mitigations would address both curtailment and voltage issues. 